\title{
Religiöse Kultur und Kommunikation
}

\author{
von Renate Köcher
}

\section{Religiöse Kultur und Kirchenbindung}

Die religiöse Kultur der Bundesrepublik hat sich in den letzten Jahrzehnten einschneidend verändert. Entscheidend waren die späten sechziger und frühen siebziger Jahre. In diesem Zeitraum brachen die kirchlichen Bindungen innerhalb nur weniger Jahre in weiten Kreisen der Bevölkerung erdrutschartig zusammen; zwischen 1973 und 1980 setzte sich der Rückzug vor allem in der jungen Generation fort, so daß sich die Generationen in religiösen Fragen immer stärker auseinanderentwickelten. Zwar gab es in den letzten Jahren keine neue Phase des erdrutschartigen Verfalls religiöser Bindungen; dennoch sinkt stetig der Anteil der Bevölkerung, der der Religion einen existentiellen Stellenwert im eigenen Leben zuschreibt und enge kirchliche Bindungen aufweist. Analysiert man zunächst die Situation für Westdeutschland, so ist der Anteil der erwachsenen Bevölkerung, der sich im weitesten Sinne zum Glauben an Gott bekennt, seit Anfang der achtziger Jahre von 72 auf $63 \%$ gesunken, der Anteil, der dem Glauben im eigenen Leben existentielle Bedeutung zuschreibt, von 44 auf $37 \%$. Auch die Intensität ihrer Gottesbeziehung, ihres Glaubens, wird heute von vielen geringer angesetzt als noch vor einem Jahrzehnt.

Parallel dazu sind das Vertrauen in die Kirchen und die Erwartung, von den Kirchen richtungweisende Antworten zu erhalten, ebenfalls gesunken. Der Kreis, der Vertrauen in die Kirchen setzt, ist von 48 auf $39 \%$ geschrumpft. Der Gottesdienstbesuch geht kontinuierlich zurück. Nur noch knapp ein Fünftel der erwachsenen Bevölkerung nimmt relativ regelmäßig an Gottesdiensten teil. Der Gottesdienst wird aus dem alltäglichen Leben der Bevölkerung immer mehr herausgedrängt und zunehmend zu einer Zeremonie, die die einschneidenden Lebensabschnitte'kennzeichnet und aus dem säkularen Alltag heraushebt. Während nur noch eine kleine Minderheit regelmäßig Gottesdienste besucht, befürwortet die überwältigende Mehrheit der westdeutschen Bevölkerung religiöse Feiern bei der Geburt eines Kindes, bei der Hochzeit und besonders bei Beerdigungen.

Der Anteil der Katholiken, die regelmäßig oder zumindest häufiger den Gottesdienst besuchen, hat sich in den letzten 20 Jahren von 40 auf $28 \%$ verringert; von den Protestanten besuchen noch $8 \%$

Dr. R. Köcher ist Geschäftsführerin des Instituts für Demoskopie, Allensbach. 
häufiger den Gottesdienst. Die Bindungen an die Kirchen lockern sich. Heute fühlen sich $31 \%$ der Katholiken, $14 \%$ der Protestanten ihrer Kirche eng verbunden; 35\% der Katholiken und 52\% der Protestanten haben nur noch schwache oder keine Bindungen. Die beiden großen Glaubensgemeinschaften sind heute sichtbar von dem Empfinden gezeichnet, die Nachhut der gesellschaftlichen Entwicklung zu stellen. Sie reagieren überwiegend defensiv auf die Veränderungen einer Gesellschaft, die sich scheinbar unaufhaltsam aus kirchlichen und religiösen Bindungen löst. Zur Zeit sind beide Konfessionsgemeinschaften weit davon entfernt, vitale, intakte religiöse Gemeinschaften zu sein.

Die häufig kolportierte These, die Erosionserscheinungen beträfen ausschließlich die Institution Kirche, nicht die Religiosität der Bevölkerung, hält der empirischen Überprüfung nicht stand. Die Zusammenhänge zwischen kirchlichen Bindungen und individueller Glaubenskraft sind unverändert denkbar eng. In den Bevölkerungskreisen mit engen kirchlichen Bindungen definieren sich $96 \%$ als religiös, von den kirchlich Distanzierten ganze $16 \%$. In der verharmlosenden Diagnose einer isolierten Kirchenkrise spiegelt sich die allgemeine Unterschätzung der Bedeutung von Institutionen. Die Faszination, die heute von Individualität und Spontaneität ausgeht, macht unempfänglich für die große Leistung, zeit- und raumübergreifend, über alle nationalen und ethnischen Grenzen hinweg, religiöse Gemeinschaft zu ermöglichen.

Die Lockerung kirchlicher Bindungen zeigt einen Verlust an religiöser Substanz an. Noch 54\% der westdeutschen, aber nur $21 \%$ der ostdeutschen Bevölkerung beschreiben sich als religiös. Durch die Vereinigung ist das zahlenmäßige Gewicht der religiös motivierten Bevölkerungskreise und der Konfessionsgemeinschaften geringer geworden. Die politische Ächtung des Religiösen, die Verdrängung des religiösen Bekenntnisses aus dem öffentlichen Raum in der ehemaligen DDR haben die kirchlichen und religiösen Bindungen unterminiert. In vielen Familien hielten der Glaube und die Bemühungen um Glaubensvermittlung der gesellschaftlichen Ächtung nicht stand. Von den unter-30jährigen Ostdeutschen wuchsen nur $8 \%$ in einem bewußt religiösen Elternhaus auf, $69 \%$ beschreiben ihr Elternhaus als völlig religionsfern. Auch in Westdeutschland ist der Anteil der in einem engagiert religiösen Elternhaus Aufgewachsenen in den letzten Jahrzehnten kontinuierlich geringer geworden. Die völlige Entfremdung von Glauben und Kirche, die weite Teile der Bevölkerung Ostdeutschlands und insbesondere der jungen Generation kennzeichnet, ist jedoch in Westdeutschland nur bei einer Minderheit anzutreffen. In Ostdeutschland bekennt sich nur noch knapp jeder dritte zum Glauben an Gott, von den Unter-30jährigen gerade noch $20 \%$, von den gleichaltrigen Westdeutschen dagegen $55 \%$.

Weniges trennt Ost- und Westdeutsche so scharf wie die kirchlichen und religiösen Bindungen. Nur 33\% der ostdeutschen Bevölkerung, aber $81 \%$ der westdeutschen Bevölkerung sind Mitglied 
einer der beiden großen Konfessionsgemeinschaften. Diese Kluft wird in den kommenden Jahren eher zunehmen als geringer werden; anders als in den alten Bundesländern sind die Konfessionsgemeinschaften in Ostdeutschland stark überaltert. Auch die konfessionelle Struktur Ostdeutschlands, wo der Protestantismus mit großem Abstand dominiert, läßt ein weiteres Auseinanderdriften der Kirchenmitgliedschaft in den alten und neuen Bundesländern erwarten. Protestanten trennen sich leichter von ihrer Kirche als Katholiken.

Die Zahl der Kirchenaustritte hat in den letzten Jahren bei beiden Konfessionsgemeinschaften ein in der Geschichte der Bundesrepublik bisher ungekanntes Ausmaß erreicht. Austrittswellen waren wiederholt zu verzeichnen, jedoch nie zuvor ein kontinuierlicher steiler Anstieg über eine so lange Zeitspanne von zehn Jahren hinweg wie zwischen 1983 und 1993. Erst 1994 trat eine Beruhigung ein. Die protestantische Kirche verzeichnet seit langem wesentlich höhere Austrittszahlen und wird auch künftig - geht man von den Absichtserklärungen von Protestanten und Katholiken aus, ihre Kirche zu verlassen - deutlich über dem Niveau der Austritte aus der katholischen Kirche liegen.

Immer wieder hat es in der Geschichte des Christentums Dürrephasen gegeben. Das Erlebnis, daß religiöse und kirchliche Bindungen für einen Teil der Gesellschaft an Bedeutung verlieren, ist jedoch ein psychologisches Problem für die religiös Gebundenen. Die Bereitschaft, auch die Fähigkeit, sich mit den eigenen Überzeugungen zu exponieren, sinkt. Untersuchungen unter Schülern zeigen, daß religiöse Schüler unter religiös desinteressierten Gleichaltrigen vermeiden, ihre Glaubensüberzeugungen anzusprechen. Das demonstrative Bekenntnis und jeglicher missionarische Eifer sind dem modernen Christentum völlig fremd. Zwar sind $42 \%$ der gesamten Bevölkerung in den letzten Jahren von jemandem offensiv und werbend auf das Thema Glauben und Kirche angesprochen worden. In fast drei Viertel der Fälle waren es jedoch Zeugen Jehovas, nur bei einer verschwindenden Minderheit Katholiken oder Protestanten, die engagiert für ihren Glauben warben.

\section{Probleme religiöser Kommunikation}

Erschwerend kommen die offensichtlichen Schwierigkeiten hinzu, über Glaubensfragen überhaupt zu sprechen. Die Kirchen und die Gläubigen kennzeichnen sich heute durch Sprachlosigkeit und Unfähigkeit, ihre Glaubensüberzeugungen darzustellen. Es ist bisher ungeklärt, wann diese Kommunikationsschwäche begann. Es deutet einiges darauf hin, $\mathrm{da}$ diese Schwierigkeiten Ende der fünfziger Jahre einsetzten, also rund ein Jahrzehnt vor dem offenkundigen Verfall kirchlicher und religiöser Bindungen. Heute dominiert die Auffassung, daß religiöse Überzeugungen in den Bereich der Intimsphäre gehören und in der Kommunikation mit anderen keinen Platz haben. Der christliche Glaube hängt jedoch 
entscheidend von der Kommunikation, von der Übermittlung ab. Zwar wird heute außerordentlich viel über die Kirche gesprochen, gerade auch in den Medien. Die Stellungnahmen und Veranstaltungen der Kirchen, die Projekte, die caritativen Aufgaben und die Kontroversen um kirchliche Normen werden ausführlich behandelt. Die religiöse Substanz der Kommunikation ist jedoch mager, Glaubensinhalte und die Bedeutung des Glaubens für den einzelnen und für die Gesellschaft werden kaum behandelt.

Die Kirchen haben heute ein Kommunikationsproblem in einem ganz umfassenden Sinn:

- Der direkte Kontakt zwischen Kirche und Konfessionsmitgliedern hat sich gelockert, die Kirchen erreichen nur noch eine Minderheit ihrer Mitglieder direkt und regelmäßig.

- Durch die Lockerung religiöser und kirchlicher Bindungen, insbesondere in der jungen Generation, nehmen Bereitschaft und Fähigkeit zur Weitergabe des Glaubens in der Kindererziehung ab. Nur noch eine Minderheit wächst in einem religiösen Elternhaus auf und erlernt dadurch mühelos die Kommunikation über religiöse Fragen.

- In der Alltagskommunikation kommen religiöse Überzeugungen kaum vor, weder in den Familien, noch in Schulen, in Beruf oder in der Freizeit.

- In den Medien wird in erster Linie über die Institution, ihre Repräsentanten, Veranstaltungen und Verlautbarungen berichtet, nicht über den individuellen Glauben. Wie in der personalen Alltagskommunikation sind Glaubensüberzeugungen auch aus der Medienkommunikation weitgehend verdrängt.

- Das säkulare Medienangebot entwickelt sich außerordentlich dynamisch, wird kontinuierlich breiter, heterogener, stärker auf Zielgruppen zugeschnitten.

- Das kirchliche Medienangebot hält mit dieser Entwicklung nicht Schritt, sondern fällt zurück.

\section{Die Probleme kirchlicher Kommunikation am Beispiel der Bistumspresse}

Die Entwicklung der deutschen Bistumszeitungen spiegelt diese Probleme. Seit 1963, als die Auflage während des Zweiten Vatikanischen Konzils mit 2,4 Millionen Exemplaren ihren Höchststand erreichte, hat sich die Auflage praktisch halbiert. Unter den Lesern sind zudem Über-60jährige, Personen mit einfacher Schulbildung und Frauen stark überrepräsentiert. Zwar ist die Bedeutung der Bistumszeitungen auch heute erheblich: Sie erreichen im weitesten Leserkreis $23 \%$ aller Katholiken ab 14 Jahre, das sind rund 4,9 Millionen; darunter zählen 2,3 Millionen zu den regelmäßigen, 2,6 Millionen zu den sporadischen Lesern. Die Entwicklung und Struktur der heutigen Leserschaft lassen jedoch den Schluß $z u$, daß ohne wesentliche Veränderungen der Bistumspresse ein anhaltender Niedergang zu erwarten ist. Die Proble- 
me der Bistumspresse haben mehrere und sehr unterschiedliche Gründe.

Die Parallelität der Entwicklungen von Bistumspresse und religiöser Kultur sind offensichtlich. Zwischen 1980 und 1994 sank der Anteil der regelmäßigen Gottesdienstbesucher unter Katholiken von 38 auf 28\%. Parallel ging der Anteil der Leser der KONPRESS-Titel um $30 \%$ zurück. Genauso sank die Auflage der Bistumspresse in diesem Zeitraum um rund $30 \%$. Mit der Lockerung der religiösen und kirchlichen Bindungen hat sich das Interesse an religiösen Themen vermindert. Der Kreis mit ausgeprägtem Interesse an religiösen Fragen ist in den alten Bundesländern seit 1967 von 31 auf 23\% zurückgegangen. Dieser Rückgang ist in beiden Konfessionen festzustellen: Unter Protestanten hat sich der Kreis, den religiöse Fragen sehr oder ziemlich interessieren, von 24 auf $17 \%$ verengt, unter Katholiken von 40 auf $32 \%$. Überdurchschnittlich ist das Interesse in der Gruppe der Unter30jährigen und der 30- bis 44jährigen zurückgegangen. Das gleiche gilt für das Interesse an kirchlichen Informationen, das sich weitgehend mit dem Interesse an religiösen Informationen deckt. Nach wie vor hängen kirchliche Bindungen und Interesse an religiösen Themen eng zusammen. Das Auseinanderdividieren von kirchlicher und religiöser Kultur, das oft vorgenommen wird, läßt sich empirisch nicht bestätigen. Von den Personen mit starken kirchlichen Bindungen interessieren sich 66\% sehr für religiöse Themen, von Personen mit schwachen kirchlichen Bindungen dagegen nur $7 \%$.

Dies hat beträchtliche Bedeutung für das Konzept der Bistumspresse: Kirchlich Gebundene sind und bleiben die primäre Zielgruppe.

Angesichts des Gleichschritts der Entwicklungen der religiösen Kultur und der Resonanz kirchlicher Medien drängt sich die Frage auf, ob nicht die Kirchenzeitungen lediglich Opfer einer Abnahme kirchlicher Bindungen sind und unabhängig von der Qualität der redaktionellen Angebote zwangsläufig an Auflage verlieren müssen, wenn sich die Entkirchlichung der Gesellschaft weiter fortsetzt.

Die Ergebnisse der Situationsanalyse der Kirchenpresse stützen jedoch die These, daß sie nicht lediglich das Opfer externer Entwicklungen sind, sondern daß auch gravierende Defizite vorliegen, die behoben werden können. ${ }^{1}$

Die Probleme der Bistumspresse gehen keineswegs ausschließlich auf die Schwächung religiöser und kirchlicher Bindungen zurück. Es gibt vielmehr weitere Ursachen, vor allem:

1 Vgl. dazu den ausführlichen Beitrag von R. Schulz, 'Chancen für die Bistumszeitungen' in diesem Heft. 
Nicht nur die Bistumspresse, sondern auch Partei- und Gewerkschaftszeitungen kämpfen zur Zeit durchgängig mit Problemen. Dies hat vor allem zwei Ursachen. Die Weltanschauungspresse hat große Mühe, in einem immer professionelleren Medienumfeld mitzuhalten. Zum zweiten trägt das Lesemotiv "Weltanschauung" alleine nicht, das heißt die Weltanschauungspresse muß über das Lesemotiv "Weltanschauung" hinaus Gratifikationen bieten, also interessant sein, Informationen bieten, die woanders so leicht nicht $\mathrm{zu}$ beziehen sind, unterhaltsam und aktuell. Das Motiv, in der eigenen weltanschaulichen, religiösen Position bestärkt zu werden, reicht alleine nicht aus.

Alle Presseorgane jenseits der aktuellen Tagespresse müssen die Frage nach dem "Zusatznutzen" beantworten, den sie über die täglich genutzten Medien Tagespresse, Fernsehen und Hörfunk hinaus bieten. Ergebnisse der Inhaltsanalyse der Bistumspresse lassen den Schluß zu, $\mathrm{daß}$ viele Bistumsblätter die Frage des Zusatznutzens für sich nicht ausreichend reflektiert haben.

\section{Das Problem der Bezugsebene}

Die Perspektive der Bevölkerung konzentriert sich auf den Nahbereich und allgemein auf religiöse Themen. Der Nahbereich ist verengt auf das persönliche Umfeld, die eigene Gemeinde. Die Bistumsebene ist keine Bezugsgröße, genauso wenig wie die Landespolitik. Die Bezugsebenen sind die Großinstitution Kirche einerseits und die Gemeinde andererseits.

\section{Die verschärfte Konkurrenz um die Zeit und die Aufmerksamkeit der Leser}

Die Bevölkerung verbringt heute im Durchschnitt $51 / 2$ Stunden pro Tag mit Medienkonsum. Davon entfallen 2 bis 3 Stunden auf Hörfunknutzung, 2 1/4 Stunden auf Fernsehkonsum und eine halbe Stunde auf die Tagespresse. Dazu kommt die Nutzung einer Fülle von Zeitschriften.

Das Medienangebot hat sich in den letzten 30 Jahren, in denen sich die Bistumspresse negativ entwickelte, vervielfacht. Heute gibt es allein 744 Zeitschriften, inklusive der Fachpresse sogar 960 Zeitschriftentitel. Durch die ständige Neugründung von Titeln und die starke Konkurrenz sinkt die Leser-Blatt-Bindung. Der Anteil regelmäßiger Leser geht bei Zeitschriften zurück, der Anteil sporadischer Leser nimmt zu.

Generell ist die Mediennutzung zunehmend von Mobilität und Flexibilität geprägt. Diese Entwicklung verstärkt den Wettbewerb, der immer mehr mit intensiver Werbung, mit Leseanreizen auf den Titelblättern und mit einer ständigen Optimierung von Aufmachung 
und Inhalten arbeitet. Die kirchlichen Medien stehen vor dem Problem, mit einem ungeheuer raschen und weiter zunehmenden Innovationstempo bei den säkularen Medien Schritt zu halten.

\section{Wachsende Bedeutung zielgruppenspezifischer Angebote}

Das Medienangebot, insbesondere im Bereich der Zeitschriften, wird immer heterogener und auf die Interessen einzelner Zielgruppen abgestellt. Insbesondere Special-Interest-Zeitschriften, die bestimmte eng gefaßte Interessen bedienen, haben außerordentlich zugenommen. Dagegen verlieren die allgemein informierenden Titel mit breiter Themenpalette mit wenigen Ausnahmen. Das Zielgruppenkonzept ist ein anderes als das der Bistumspresse. Kirchliche Bindungen und Interesse an religiösen Fragen definieren keine eng gefaßte, in sich geschlossene Zielgruppe, sondern ein sehr heterogenes Publikum.

$V$. Inhalte und Aufmachung der kirchlichen Kommunikation, speziell auch der Bistumspresse, sind der Konkurrenz der säkularen Medien unzureichend gewachsen

Die Veränderungen des Informations- und Kommunikationsverhaltens und der Wandel der Leserbedürfnisse werden von vielen kirchlichen Medien nur unzureichend aufgenommen. Dies zeigen Ergebnisse von Inhaltsanalysen wie von Leserbefragungen. Es spricht viel dafür, daß die Chancen der Bistumspresse unzureichend genutzt werden wie auch die Chancen des religiösen Buchs, für das beträchtliche, unausgeschöpfte Potentiale bestehen. Immerhin ein Fünftel der gesamten Bevölkerung zählt zu den religiös interessierten Buchkäufern, das sind rund 12 Millionen Menschen.

Der gesamte Bereich der kirchlichen und religiösen Kommunikation hat nicht nur ein Umfeldproblem, ist nicht nur Opfer der gesellschaftlichen Entwicklung, sondern hat in weiten Teilen auch interne Organisations-, Personal- und Qualitätsprobleme.

Es wird viel davon abhängen, wieweit es gelingt, den Bereich der kirchlichen und religiösen Kommunikation stärker zu professionalisieren und an ein verändertes Umfeld, das wesentlich höhere Anforderungen an Medien stellt, anzupassen. Die Chancen, in der Gesellschaft präsent zu sein, Menschen zu erreichen, eigene Positionen und Werte zu vermitteln, für dèn Glauben zu werben, hängen heute in hohem Maße von der Qualität der medialen Kommunikation ab. Wer den Kommunikationsbereich vernachlässigt, vergibt Chancen, diese Gesellschaft zu prägen. 


\section{SUMMARY: Religious culture and communication}

At the beginning, the article lists some actual statistics on the declining of religious and Church life in the Federal Republic of Germany since the late $1960 \mathrm{~s}$. Then the attention is called to the fact that the increasing number of people cancelling their Church membership is not a mere problem of the Churches as institutions but is - contrary to frequent speculations - connected with a real loss of religiosity in society. On this background, the author points out some problems and perspectives of Church press including difficulties in administration, qualified personel and lacking quality in the papers.

\section{RÉSUMÉ: Culture religieuse et communication}

L'article nous livre tout d'abord quelques chiffres récents sur le recul constant de la religiosité et de ce qui est religieux en Allemagne Fédérale depuis la fin des années 60. 11 attire notre attention sur le fait que le nombre croissant de personnes qui se retire de l'église ne représente pas seulement un problème pour les églises en tant qu' institution, mais - contrairement à une supposition souvent exprimée -- que cela est lié à une réelle perte de religiosité. En ne perdant pas de vue ce fait, l'auteur mentionne quelques problèmes et perspectives de la presse religieuse qui a surtout des problèmes internes relatifs a l'organisation, au personnel et à la qualité.

\section{RESUMEN: Cultura religiosa y comunicación}

El artículo nos oferece primeramente algunas cifras actuales referentes a la continua disminución de la religiosidad y devoción en la RFA desde finales de los años 60 . Hace hincapié que el aumento del número de las bajas de la iglesia no es solamente un problema de las iglesias como instituciónes, sino que, al contrario de la idea que a menudo se tiene, éste se encuentra ligado a una pérdida real de la religiosidad. En este contexto, la autora menciona varios problemas y perspectivas de la prensa de la iglesia, que, en diversas partes, tiene problemas internos de organización, de personal y de calidad. 